\section{Case Reports in Oncology}

\title{
Clinical Benefit from Trametinib in a Patient with Appendiceal Adenocarcinoma with a GNAS R201H Mutation
}

\author{
Celina Ang ${ }^{a}$ Aryeh Stollman $^{b}$ Hongfa Zhu ${ }^{c}$ Umut Sarpel ${ }^{d}$ \\ Bethann Scarborough ${ }^{e}$ Gagan Sahni ${ }^{f}$ Sherri Z. Millis ${ }^{9}$ \\ ${ }^{a}$ Division of Hematology/Oncology, Icahn School of Medicine at Mount Sinai, \\ New York, NY, USA; ${ }^{b}$ Department of Radiology, Icahn School of Medicine at Mount Sinai, \\ New York, NY, USA; ${ }^{\circ}$ Department of Anatomic Pathology, Icahn School of Medicine at \\ Mount Sinai, New York, NY, USA; ${ }^{d}$ Department of Surgery, Division of Surgical Oncology, \\ Icahn School of Medicine at Mount Sinai, New York, NY, USA; ${ }^{\mathrm{B}}$ Brookdale Department \\ of Geriatrics and Palliative Medicine, Icahn School of Medicine at Mount Sinai,

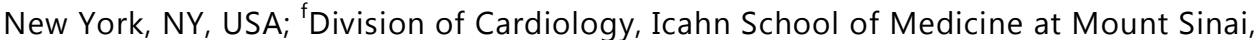 \\ New York, NY, USA; ${ }^{9}$ Foundation Medicine, Inc., Cambridge, MA, USA
}

\section{Keywords}

Appendiceal adenocarcinoma - Targeted therapy $\cdot$ GNAS mutation · Trametinib

\begin{abstract}
We report the case of a patient with appendiceal adenocarcinoma with mucinous peritoneal carcinomatosis who was treated with trametinib upon identification of a GNAS R201H mutation by comprehensive genomic profiling. The molecular pathology of appendiceal neoplasms is reviewed, and the mechanistic basis underlying the clinical benefit as well as the subsequent course on trametinib that were observed in this patient are discussed.
\end{abstract}




\section{Case Reports in Oncology}

\section{Introduction}

Appendiceal neoplasms are rare, occurring in $<1.5 \%$ of appendectomy specimens [1]. Histologic variants include adenocarcinomas, low- and high-grade mucinous neoplasms, goblet cell carcinoids, and neuroendocrine tumors [1, 2]. Pseudomyxoma peritonei (PMP) results from peritoneal dissemination of mucinous appendiceal neoplasms [2]. Surgery is indicated for early-stage disease, and in patients with PMP, aggressive cytoreductive surgery with hyperthermic intraperitoneal chemotherapy can yield durable disease control [3]. For unresectable or metastatic appendiceal adenocarcinoma, systemic therapy following colorectal paradigms is the mainstay, though clinical trial data are limited [4]. Comprehensive genomic profiling (CGP) offers the promise of personalized therapy and may yield clinical benefit when standard chemotherapies are no longer effective. We present a patient with a mucinous appendiceal adenocarcinoma and PMP refractory to several conventional chemotherapy agents who experienced clinical benefit from trametinib based on CGP results demonstrating a GNAS R201H mutation.

\section{Case Presentation}

A 53-year-old man previously in good health developed abdominal distension. Computed tomography (CT) showed a dilated appendix measuring $9.4 \times 4.4 \mathrm{~cm}$ and large-volume gelatinous ascites with extensive peritoneal carcinomatosis measuring up to $3.0 \mathrm{~cm}$ in thickness, consistent with PMP from a mucinous appendiceal neoplasm. Cytoreductive surgery and hyperthermic intraperitoneal chemotherapy were attempted but aborted as optimal debulking could not be achieved. Peritoneal cytology and omental specimens revealed a disseminated well-differentiated mucinous adenocarcinoma expressing CK20, CDX-2, MLH1, MSH2, MSH6, and PMS2, and wild-type KRAS and BRAF. The patient started FOLFIRINOX chemotherapy given his age and good functional status. Baseline serum CA 19-9 and CEA were $449.8 \mathrm{U} / \mathrm{mL}$ and $3.1 \mathrm{ng} / \mathrm{mL}$, respectively. His disease remained stable on therapy for 6 months, with a slight decrease in ascites and a decrease in CA $19-9$ to $226.5 \mathrm{U} / \mathrm{mL}$ and in CEA to $2.0 \mathrm{ng} / \mathrm{mL}$. CA 125 , not previously checked, was $29 \mathrm{U} / \mathrm{mL}$. Due to worsening oxaliplatin neuropathy he switched to FOLFIRI + bevacizumab for 6 months. Repeat CT of the chest, abdomen, and pelvis showed an $8.4 \times 4.6 \mathrm{~cm}$ appendiceal mass and a $2.6 \mathrm{~cm}$ peritoneal carcinomatosis. However, his CA 19-9 increased acutely to 4,730.6 U/mL, CA 125 to $64 \mathrm{U} / \mathrm{mL}$, and CEA to $6.4 \mathrm{ng} / \mathrm{mL}$. Given the cumulative side effects from treatment and absence of disease-related symptoms, following extensive discussion, the patient took a 2-month break from chemotherapy. In the interim his CA 19-9 and CEA rose to 7,805.5 $\mathrm{U} / \mathrm{mL}$ and $20.5 \mathrm{ng} / \mathrm{mL}$, respectively, though his disease remained grossly stable radiographically. Irinotecan + panitumumab was started, but his disease progressed after 3 months with a commensurate rise in CA $19-9$ to $8,463.7 \mathrm{U} / \mathrm{mL}$ and a slight decrease in CEA to $17.9 \mathrm{ng} / \mathrm{mL}$. On CT, the appendiceal mass was stable and the peritoneal thickness was $1.8 \mathrm{~cm}$, but the extent of carcinomatosis had increased, and the patient required admission for pain and management of ascites. A large-volume paracentesis was unsuccessful because of extensive loculation. An intraperitoneal catheter was subsequently placed, draining approximately $100 \mathrm{~mL}$ of serosanguinous fluid daily. Given continued progression after 18 months of standard chemotherapy, archived omental tissue was submitted to a CLIAcertified, CAP-accredited laboratory (Foundation Medicine, Inc., Cambridge, MA) for CGP. This revealed an activating GNAS R201H mutation. Though no clinical studies have directly 


\section{Case Reports in Oncology}

linked GNAS mutations in cancer to targeted therapies, preclinical evidence suggests that tumors with GNAS mutations may be sensitive to MAPK and Wnt pathway inhibitors [5]. Trametinib is an FDA-approved noncompetitive inhibitor of ATP which binds MEK adjacent to the ATP binding site, similar to other MEK allosteric inhibitors, thus inhibiting RAFdependent phosphorylation of MEK1 [6]. It was thus decided to treat the patient with the MEK inhibitor trametinib $2 \mathrm{mg}$ daily.

The patient underwent a baseline ophthalmologic assessment and radionuclide ventriculography which showed normal biventricular size and function as well as a left ventricular ejection fraction (LVEF) of 50\%. Within the first 2 weeks of therapy, he developed a grade 2 acneiform rash affecting the face, upper chest, and back, as well as lower extremity edema. Drainage from his peritoneal catheter slowly decreased and eventually ceased. After 2 months, his CA $19-9$ had decreased to $4,428.7 \mathrm{U} / \mathrm{mL}$, CEA to $6.5 \mathrm{ng} / \mathrm{mL}$, and CA 125 to $38 \mathrm{U} / \mathrm{mL}$. On repeat CT, his appendiceal mass was $7.4 \times 4.5 \mathrm{~cm}$ and the peritoneal carcinomatosis was stable. Although his peritoneal catheter had stopped draining 2 months earlier, his abdominal girth remained stable without reaccumulation of ascites. His analgesic requirements also decreased from MS Contin $30 \mathrm{mg}$ every $8 \mathrm{~h}$ and morphine $10 \mathrm{mg}$ every $4 \mathrm{~h}$ at the start of trametinib to morphine $10 \mathrm{mg}$ prn. Repeat echocardiography performed because of persistent lower extremity edema showed a decrease in LVEF to $40 \%$ which was attributed to trametinib since his pretreatment LVEF had been $50 \% 3$ months earlier. After discussion of the risks and benefits of continuing trametinib in light of his cardiac dysfunction and considering the fact that he was asymptomatic apart from edema, he continued trametinib at a reduced dose of $1 \mathrm{mg}$ daily. Given stability of his symptoms and LVEF 1 month later, trametinib was increased to $1.5 \mathrm{mg}$ daily. Tumor markers remained stable 3.5 months after starting trametinib: CA $19-9$ was $4,624.5 \mathrm{U} / \mathrm{mL}$, CEA was $6.2 \mathrm{ng} / \mathrm{mL}$, and CA 125 was $21 \mathrm{U} / \mathrm{mL}$. One month later, he was admitted for persistent nausea, vomiting, and poor oral intake. Repeat imaging demonstrated a partial small bowel obstruction but no significant change in overall disease burden. He was evaluated by gastroenterology and surgical oncology, but his disease was not amenable to stent placement nor venting gastrostomy. After extensive discussions, the patient decided to discontinue therapy and return to his home country.

\section{Discussion}

The molecular topography of mucinous appendiceal neoplasms is characterized by KRAS mutations in $40-78 \%$ of cases and by alterations in APC, ATM, PIK3CA, SMAD4, NRAS, GNAS, TP53, and the TGF- $\beta$ pathway, particularly in adenocarcinomas [7, 8]. GNAS mutations are primarily the hallmark of low-grade appendiceal mucinous neoplasms (24-82\%), but have also been reported in low-grade/well-differentiated adenocarcinomas (22-35\%) and in high-grade adenocarcinomas with (15\%) and without signet rings (37\%) [7-12]. GNAS encodes the alpha subunit of the stimulatory G protein (Gs-alpha), a guanine-nucleotide binding protein (G protein) involved in hormonal regulation of adenylate cyclase [13]. $R 201 H$ is an activating mutation causing increased Gnas/XL $\alpha$ s activity, increased cAMP accumulation, and constitutive cAMP signaling, associated with excessive proliferation and tumor development $[5,13,14]$. Clinically, GNAS activation may be responsible for the copious mucin production associated with PMP [10]. The demographics, presence or absence of adverse pathologic features, and survival outcomes of patients with appendiceal neoplasms do not appear to be impacted by GNAS mutation status [11,12]. 
Since GNAS activation may affect downstream MAPK and Wnt signaling pathways [5], inhibitors of these pathways, such as trametinib, may be relevant to tumors with GNASactivating mutations.

Our patient experienced meaningful, albeit short-lived, clinical benefit - including an improvement in quality of life - from trametinib, suggesting that targeted MAPK pathway inhibition was important in inducing tumor response. His time to progression of about 4 months on trametinib is comparable to the median progression-free survival of 4.8 months reported in patients with $B R A F$-mutated melanoma [15]. The dose reduction in trametinib because of cardiotoxicity prompts speculation about whether this might have shortened the duration of his clinical response. Based on preclinical data, the target plasma trametinib concentration for MEK pathway inhibition is $10.4 \mathrm{ng} / \mathrm{mL}[15,16]$. Trametinib $2 \mathrm{mg}$ daily exceeds this target concentration throughout the dosing period, whereas a 1-mg daily dose yields concentrations that mostly remain below this threshold [17]. Pharmacokinetic analysis from a phase 2 study of trametinib reported a shorter progression-free survival in patients with median plasma trametinib concentrations $<10.6 \mathrm{ng} / \mathrm{mL}$ than in those with concentrations $\geq 10.6 \mathrm{ng} / \mathrm{mL}$ [15].

To our knowledge, this is the first case of an appendiceal mucinous neoplasm treated with trametinib for GNAS alteration, highlighting the clinical potential of personalized, targeted therapy in rare malignancies.

\section{Statement of Ethics}

The authors have no ethical conflicts to disclose.

\section{Disclosure Statement}

S.Z.M. is an employee of Foundation Medicine, Inc. The other authors have no conflicts of interest to disclose.

\section{References}

1 Turaga KK, Pappas SG, Gamblin T: Importance of histologic subtype in the staging of appendiceal tumors. Ann Surg Oncol 2012;19:1379-1385.

-2 Carr NJ, Cecil TD, Mohamed F, et al: A consensus for classification and pathologic reporting of pseudomyxoma peritonei and associated appendiceal neoplasia: the results of the Peritoneal Surface Oncology Group International (PSOGI) modified Delphi process. Am J Surg Pathol 2016;40:14-26.

-3 Verwaal VJ, Bruin S, Boot H, et al: 8-year follow-up of randomized trial: cytoreduction and hyperthermic intraperitoneal chemotherapy versus systemic chemotherapy in patients with peritoneal carcinomatosis of colorectal cancer. Ann Surg Oncol 2008;15:2426-2432.

-4 Tejani MA, ter Veer A, Milne D, Ottesen R, Bekaii-Saab T, Benson AB 3rd, Schrag D, Shibata S, Skibber J, Weiser M, Wilkinson N, Cohen SJ: Systemic therapy for advanced appendiceal adenocarcinoma: an analysis from the NCCN Oncology Outcomes Database for colorectal cancer. J Natl Compr Canc Netw 2014;12:1123-1130.

5 Wilson CH, McIntyre RE, Arends MJ, Adams DJ: The activating mutation R201C in GNAS promotes intestinal tumourigenesis in Apc(Min/+) mice through activation of Wnt and ERK1/2 MAPK pathways. Oncogene 2010;29:4567-4575.

-6 Gilmartin AG, Bleam MR, Groy A, et al: GSK1120212 (JTP-74057) is an inhibitor of MEK activity and activation with favorable pharmacokinetic properties for sustained in vivo pathway inhibition. Clin Cancer Res 2011;17:989-1000. 
7 Liu X, Mody K, de Abreu FB, et al: Molecular profiling of appendiceal epithelial tumors using massively parallel sequencing to identify somatic mutations. Clin Chem 2014;60:1004-1011.

-8 Noguchi R, Yano H, Gohda Y, et al: Molecular profiles of high-grade and low-grade pseudomyxoma peritonei. Cancer Med 2015;4:1809-1816.

-9 Hara K, Saito T, Hayashi T, et al: A mutation spectrum that includes GNAS, KRAS and TP53 may be shared by mucinous neoplasms of the appendix. Pathol Res Pract 2015;211:657-664.

$\rightarrow 10$ Alakus H, Babicky ML, Ghosh P, et al: Genome-wide mutational landscape of mucinous carcinomatosis peritonei of appendiceal origin. Genome Med 2014;6:43.

11 Nishikawa G, Sekine S, Ogawa R, Matsubara A, Mori T, Taniguchi H, Kushima R, Hiraoka N, Tsuta K, Tsuda H, Kanai Y: Frequent GNAS mutations in low-grade appendiceal mucinous neoplasms. Br J Cancer 2013;108:951-958.

12 Singhi AD, Davison JM, Choudry HA, et al: GNAS is frequently mutated in both low-grade and high-grade disseminated appendiceal mucinous neoplasms but does not affect survival. Hum Pathol 2014;45: $1737-1743$

13 Mariot V, Wu JY, Aydin C, et al: Potent constitutive cyclic AMP-generating activity of XLas implicates this imprinted GNAS product in the pathogenesis of McCune-Albright syndrome and fibrous dysplasia of bone. Bone 2011;48:312-320.

14 Landis CA, Masters SB, Spada A, et al: GTPase inhibiting mutations activate the alpha chain of Gs and stimulate adenylyl cyclase in human pituitary tumours. Nature 1989;340:692-696.

-15 Flaherty KT, Infante JR, Daud A, et al: Combined BRAF and MEK inhibition in melanoma with BRAF V600 mutations. N Engl J Med 2012;367:1694-1703.

-16 Ouellet D, Kassir N, Chiu J, Mouksassi MS, Leonowens C, Cox D, DeMarini DJ, Gardner O, Crist W, Patel K: Population pharmacokinetics and exposure-response of trametinib, a MEK inhibitor, in patients with BRAF V600 mutation-positive melanoma. Cancer Chemother Pharmacol 2016;77:807-817.

$\checkmark 17$ Infante JR, Fecher LA, Falchook GS, et al: Safety, pharmacokinetic, pharmacodynamic, and efficacy data for the oral MEK inhibitor trametinib: a phase 1 dose-escalation trial. Lancet Oncol 2012;13:773-781. 